\title{
PGC-1 $\alpha$ integrates glucose metabolism and angiogenesis in multiple myeloma cells by regulating VEGF and GLUT-4
}

\author{
DEDONG CAO*, HAO ZHOU* ${ }^{*}$ JIKAI ZHAO, LU JIN, WEN YU, HAN YAN, YU HU and TAO GUO \\ Department of Hematology, Union Hospital, Tongji Medical College, Huazhong University of \\ Science and Technology, Wuhan, Hubei 430000, P.R. China
}

Received November 8, 2013; Accepted December 16, 2013

DOI: $10.3892 /$ or.2014.2974

\begin{abstract}
Human peroxisome proliferator-activated receptor- $\gamma$ coactivator $1 \alpha$ (PGC-1 $\alpha)$ is a key coactivator in the regulation of gene transcriptional activity in normal tissues. However, it is not clear whether it is involved in the angiogenesis and metabolism of multiple myeloma (MM). The aim of the present study was to investigate the role of PGC-1 $\alpha$ in MM. Small interfering RNA (siRNA) was used to inhibit PGC-1 $\alpha$ expression in RPMI-8226 cells. An endothelial cell migration assay was performed using Transwell chambers and the expression of PGC-1 $\alpha$, estrogen-related receptor- $\alpha$ (ERR- $\alpha$ ), vascular endothelial growth factor (VEGF) and glucose transporter-4 (GLUT-4) was tested by reverse transcriptionpolymerase chain reaction (RT-PCR). The protein expression of PGC-1 $\alpha$, ERR- $\alpha$ and GLUT- 4 was assayed by western blot analysis. Lastly, RPMI-8226 cell proliferation was evaluated using CCK-8 assay. VEGF and GLUT-4 mRNA levels were decreased in cells treated with siRNA targeting PGC-1 $\alpha$, as was the level of GLUT-4 protein. Endothelial cell migration was significantly reduced when these cells were cultured with culture medium from RPMI- 8226 cells treated with siPGC- $1 \alpha$. The proliferation rates at 24 and $48 \mathrm{~h}$ were suppressed by PGC-1 $\alpha$ inhibition. Our results showed that inhibition of PGC-1 $\alpha$ suppresses cell proliferation probably by downregulation of VEGF and GLUT-4. The present study suggests that PGC-1 $\alpha$ integrates angiogenesis and glucose metabolism in myeloma through regulation of VEGF and GLUT-4.
\end{abstract}

\section{Introduction}

Multiple myeloma (MM) accounts for nearly $20 \%$ of mortality due to total hematologic tumors (1). The pathologic features

Correspondence to: Dr Tao Guo, Department of Hematology, Union Hospital, Tongji Medical College, Huazhong University of Science and Technology, Wuhan, Hubei 430000, P.R. China

E-mail: guotao1968@163.com

*Contributed equally

Key words: multiple myeloma, PGC-1 $\alpha$, VEGF, GLUT-4 of MM involve aberrant activation of metabolism and signal pathways, including uncontrolled and unlimited angiogenesis and increased glucose consumption. These changes play important roles in the clinical course of MM, particularly MM invasion and metastasis (2-4).

The angiogenic switch is controlled by the activation of pro-tumor genes such as hypoxia inducible factor-1 (HIF-1) and brain-derived neurotrophic factor (BDNF), which encode pro-angiogenic factors, such as vascular endothelial growth factor (VEGF) (5-7). Studies have shown that angiogenesis is associated with upregulated HIF/VEGF pathways in $~ 40 \%$ MM patients (6), suggesting that other pathways could be involved in the regulation of VEGF besides the HIF/VEGF pathway.

PGC-1 $\alpha$ is an important co-activator that participates in the regulation of gene transcriptional activity (8). It is now well established that PGC-1 $\alpha$ contributes to several important functions, including mitochondrial biogenesis and metabolism, by interacting with transcription factors such as nuclear respiratory factors(NRFs) and estrogen-related receptor (ERR)- $\alpha$ (9-11). PGC-1 $\alpha$ influences glucose consumption by regulating glucose transporter-4 (GLUT-4), which is upregulated in $\mathrm{MM}$ and is responsible for basal glucose consumption and maintenance of myeloid cell leukemia-1 (Mcl-1) expression, growth and survival (3). Studies have also found that PGC-1 $\alpha$ promotes angiogenesis by binding with ERR- $\alpha$, thereby increasing VEGF expression (12). Thus, angiogenesis and metabolism may be linked via PGC- $1 \alpha$. However it is not clear whether PGC-1 $\alpha$ is involved in the regulation of angiogenesis and glucose metabolism in MM. Therefore, in the present study, we explored the role of PGC- $1 \alpha$ in angiogenesis and glucose metabolism in MM.

\section{Materials and methods}

Cells and culture. Chemicals were purchased from HyClone, Sigma and Thermo Fisher Scientific, unless otherwise noted. The human MM cell line RPMI-8226 was obtained from the American Type Culture Collection (ATCC; Manassas, VA, USA) and was grown in RPMI-1640 medium (HyClone, Thermo Fisher Scientific, Waltham, MA, USA) supplemented with $10 \%$ fetal bovine serum (FBS) and $100 \mathrm{U} / \mathrm{ml}$ penicillin/streptomycin. Human umbilical vein endothelial cells (HUVECs) were cultured using the trypsin digestion method. 
All cells were grown at $37^{\circ} \mathrm{C}$ in an atmosphere containing $5 \%(\mathrm{v} / \mathrm{v}) \mathrm{CO}_{2}$. Peripheral blood mononuclear cells from normal healthy volunteers were separated and used as the normal control.

Cell proliferation assay with $C C K-8$ reagent. Cell proliferation was assayed using Cell Counting Kit-8 (CCK-8) according to the manufacturer's protocol (Dojindo Laboratories, Kumamoto, Japan). Cells were suspended at a concentration of $5 \times 10^{4} / \mathrm{ml}$ in complete medium, seeded in 96 -well plates at $0.1-\mathrm{ml}$ suspension/well, and cultured at $37^{\circ} \mathrm{C}$. Then, $10 \mu \mathrm{l}$ CCK-8 solution was added to each well after 24 and $48 \mathrm{~h}$ of culture, respectively. After incubation at $37^{\circ} \mathrm{C}$ for $1 \mathrm{~h}$, the plate was examined with a microplate reader (Bio-Rad, La Jolla, CA, USA) and the absorbance at $450 \mathrm{~nm}$ was recorded. Each experiment was performed in triplicate.

siRNA transfection. siRNA duplexes for ERR- $\alpha$ and PGC-1 $\alpha$ were designed and produced by Shanghai GenePharma Co., Ltd. (Shanghai, China). RPMI-8226 cells were transfected with Lipofectamine ${ }^{\circledR} 2000$ reagent (Invitrogen, Carlsbad, CA, USA) according to the manufacturer's protocol. The sequence information for siRNA was: siERR- $\alpha, 5$ '-GGCAGAAA CCUAUCUCAGGUU-3' (sense) and 5'-CCUGAGAUAG GUUUCUGCCUC-3' (antisense); siPGC-1 $\alpha, 5$ '-GCCAAA CCAACAACUUUAUUU-3' (sense) and 5'-AUAAAGUU GUUGGUUUGGCUU-3' (antisense).

Migration assay. Endothelial cell migration was assessed as previously described (13). Briefly, 1.5-2.0x104 of RPMI-8226 cells were loaded in the lower chamber of Transwell, and siRNA targeting PGC-1 $\alpha$ or ERR- $\alpha$ was added. After 4-6 h of transfection, the culture medium was replaced with fresh RPMI-1640 medium, and then cultured for $24 \mathrm{~h}$. Then, 1.5-2.0x $10^{4}$ of HUVECs were seeded in the upper chamber (8- $\mu \mathrm{m}$ pore; Costar Corp., Cambridge, MA, USA). The plates were then cultured at $37^{\circ} \mathrm{C}$ in $5 \% \mathrm{CO}_{2}$ for $12 \mathrm{~h}$. The cells migrated across the membrane and adhered to the lower part of the membrane, while those that did not migrate were removed with a cotton swab. The former were stained with crystal violet and examined under a microscope. The cell number before and after the experiments was counted in order to quantify proliferation.

RT-PCR. Total RNA was extracted using the TRIzol-based method (Sigma) from RPMI-8226 cells and the biopsy samples. Approximately $2 \mu \mathrm{g}$ of total RNA was reverse-transcribed into the first-strand complementary DNA (cDNA) pool using a First-Strand cDNA Synthesis kit and real-time RT-PCR was carried out using SYBR-Green (Toyobo Co., Ltd., Osaka, Japan) with the Applied Biosystems 7500 System (Life Technologies, Carlsbad, CA, USA) according to the manufacturer's instructions. Data analysis was carried out using the comparative $\mathrm{Ct}$ method. The following human-specific primers were used: $\beta$-actin, 5'-TTCCAGCCTTCCTTCCTGG-3' (forward) and 5'-TTGCGCTCAGGAGCAAT-3' (reverse); PGC-1 $\alpha$, 5'-TGGTGCCACCACCATCAAAGA-3' (forward) and 5'-TC ACCAAACAGCCGCAGACTG-3' (reverse); ERR- $\alpha, 5^{\prime}$-GTG GATGGAGGTGCTGGTGCT-3' (forward) and 5'-AGCCT CGGCATCTTCGATGTG-3' (reverse); MEF2C, 5'-GCCCT

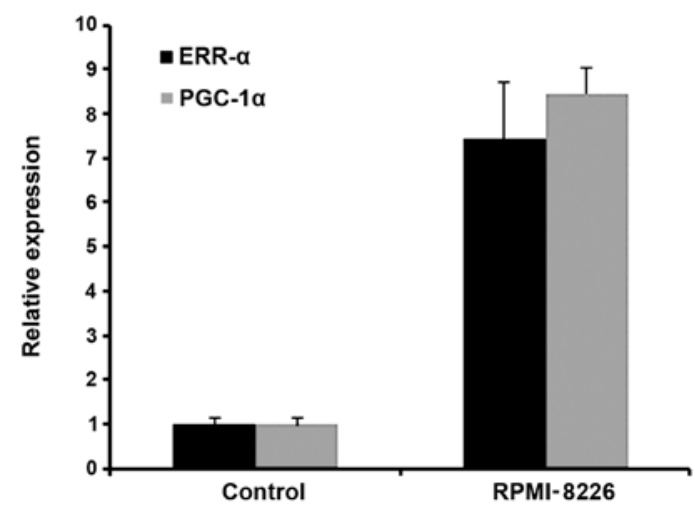

Figure 1. Relative expression of PGC- $1 \alpha$ and ERR- $\alpha$ in RPMI-8226 cells mRNA of PGC- $1 \alpha$ and ERR- $\alpha$ is present in RPMI-8226 cells and $>7$-fold higher than that of peripheral blood mononuclear cells from normal healthy volunteers. Levels are expressed as percentage relative to the values obtained for control cells.

GAGTCTGAGGACAAG-3' (forward) and 5'-AGTGAG CTGACAGGGTTGCT-3' (reverse); GLUT-4, 5'-GGCTTC TTCATCTTCACCTTCT-3' (forward) and 5'-CTCAGTTC TGTGCTGGGTTTC-3' (reverse); VEGF, 5'-GAAGTGGTG AAGTTCATGGATGTC-3' (forward) and 5'-CGATCGTTC TGTATCAGTCTTTCC-3' (reverse).

Western blot analysis. Total cell proteins were prepared, fractionated and electroblotted on sodium dodecyl sulfate gels, and western blot analysis was performed as previously described (12).

Statistical analysis. To measure overall differences, particularly those between different treatments and control, SPSS 17.0 (SPSS Inc., Chicago, IL, USA) was used. Analysis of variance and post-hoc tests (two-sided Dunnett's t test) were applied to analyze the average values of replicate results obtained by independent experiments. A P-value of $<0.05$ was considered to indicate a statistically significant difference.

\section{Results}

$P G C-1 \alpha$ is upregulated in MM. We found that the level of PGC-1 $\alpha$ was upregulated in the MM RPMI- 8226 cells. The level of ERR- $\alpha$, which is a PGC- $1 \alpha$ related co-activated factor, was also increased. The relative expression of PGC-1 $\alpha$ and ERR- $\alpha$ in RPMI-8226 cells was $>7$-fold higher than that in the control (Fig 1).

$P G C-1 \alpha$ regulates the expression of VEGF in vitro. The effect of PGC- $1 \alpha$ and ERR- $\alpha$ on the expression of VEGF in the MM cell line RPMI-8226 was examined. PGC-1 $\alpha$ was suppressed by siRNA in the RPMI-8226 cells, and the VEGF mRNA expression was then measured by RT-PCR. As shown in Fig. 2, our results showed that VEGF mRNA was significantly lower in the siPGC-1 $\alpha$ group than in the control. Next, we treated the RPMI-8226 cells with siRNA targeting ERR- $\alpha$ and found that mRNA level of VEGF was also markedly reduced. These data indicated that both PGC- $1 \alpha$ and ERR- $\alpha$ were required for the regulation of VEGF in RPMI-8226 cells, and these results are in accordance with those of other studies (12). 


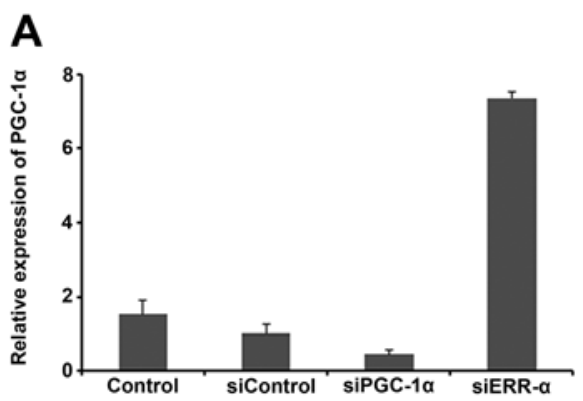

D

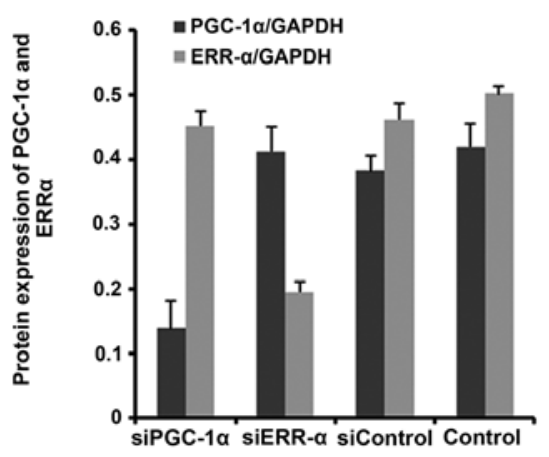

\section{B}

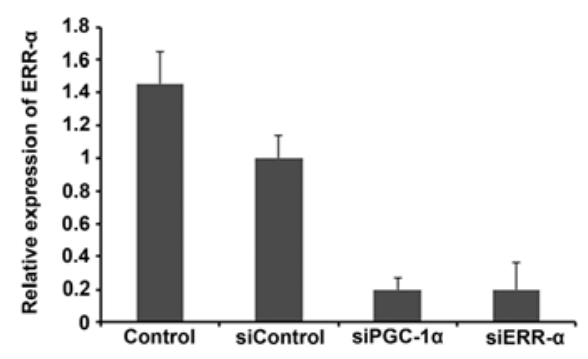

C

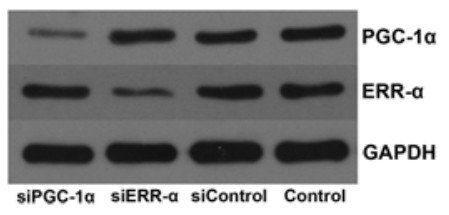

$\mathbf{E}$

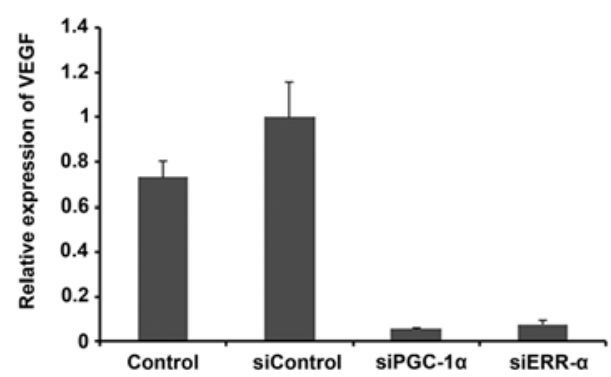

Figure 2. (A-D) PGC-1 $\alpha$ and ERR- $\alpha$ expression in RPMI-8226 cells treated with siRNA. (A and B) mRNA and (C and D) protein expression of PGC-1 $\alpha$ and ERR- $\alpha$ in RPMI-8226 cells were downregulated after siPGC- $1 \alpha$ and siERR- $\alpha$ treatment; (E) VEGF expression in RPMI-8226 cells treated with siRNA. After the RPMI-8226 cells were transfected with siRNA, they showed suppressed VEGF expression in RPMI-8226 cells when compared with the siControl group, respectively.
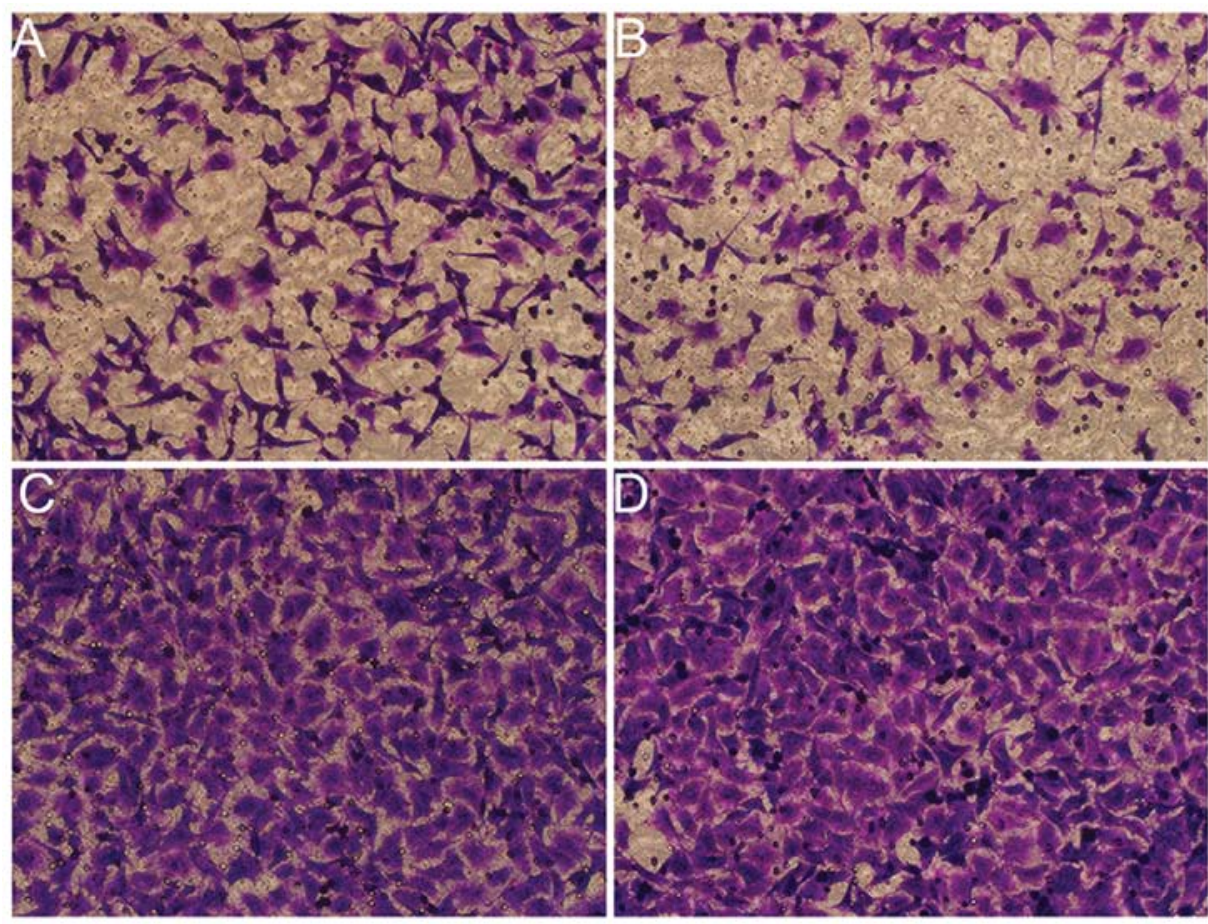

Figure 3. In vitro angiogenesis. From top left above to bottom right: (A) siPGC-1 $\alpha$, (B) siERR- $\alpha$, (C) siCONTROL and (D) control group. Migration of HUVECs in different treatment groups. The migration of HUVECs was reduced when co-cultured with RPMI-8226 cells transfected with siPGC-1 $\alpha$ or siERR- $\alpha$ (A and B), whereas migration of HUVECs was unchanged compared with the control group (C and D).

Suppression of $P G C-1 \alpha$ inhibits in vitro angiogenesis in RPMI-8226 cells. In the present study, we also determined whether PGC-1 $\alpha$ and ERR- $\alpha$ inhibition of MM cells affects the migration of human vascular endothelial cells. As shown in Fig. 3, RPMI-8226 cells without any treatment evidently promoted the migration of HUVECs, wherein a large number 

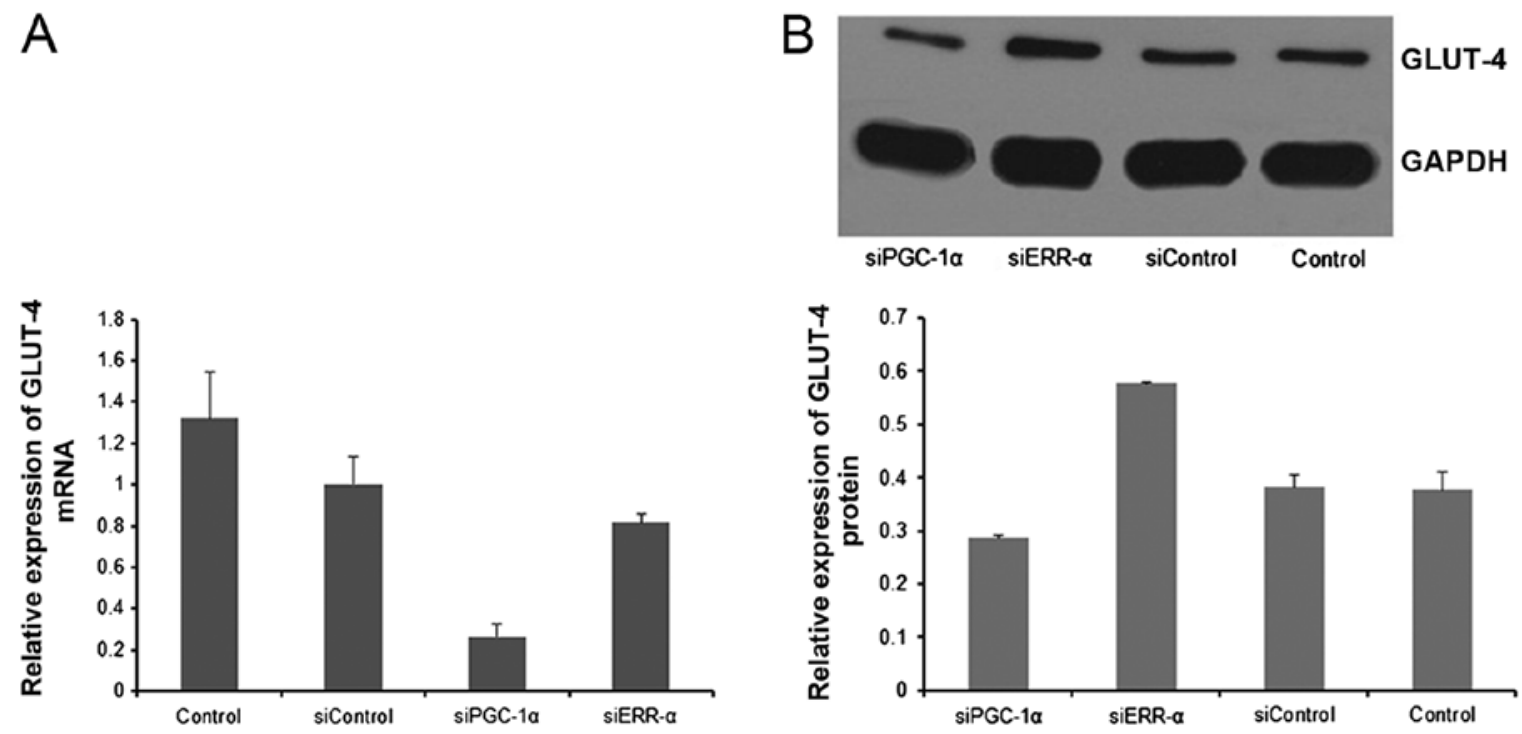

Figure 4. (A) Expression of GLUT-4 mRNA in RPMI-8226 cells. (B) Relative expression of GLUT-4 protein in RPMI-8226 cells. GLUT-4 expression was reduced when inhibiting the expression of PGC-1 $\alpha$ in RPMI-8226 cells by siRNA. GLUT-4 mRNA expression was decreased in siPGC-1 $\alpha$-transfected RPMI- 8226 cells, accompanied by a modest decrease in the level of the GLUT-4 protein. In cells treated with siERR- $\alpha$, a $45 \%$ increase of GLUT- 4 expression was observed compared with the siControl group.

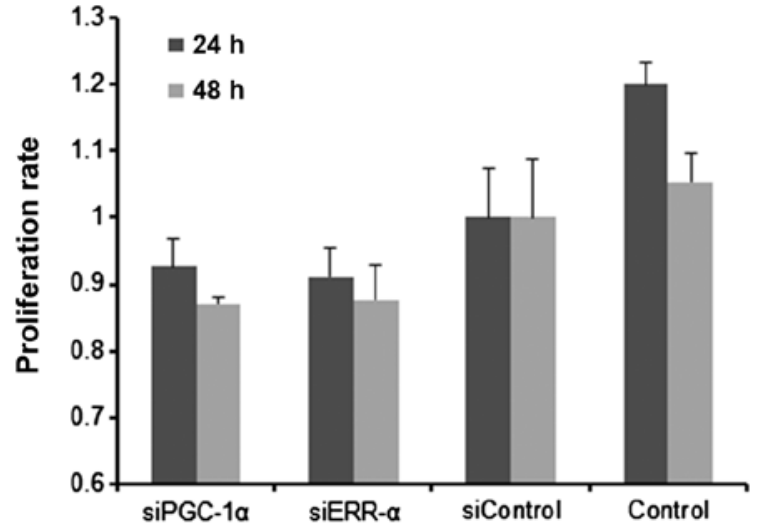

Figure 5. Proliferation rate at 24 and $48 \mathrm{~h}$. We evaluated the effects of inhibiting PGC-1 $\alpha$ or ERR- $\alpha$ on the proliferation of RPMI-8226 cells. RPMI-8226 cells were transiently transfected with siPGC-1 $\alpha$, siERR- $\alpha$ or the negative control without specifically targeting any human gene products. A cell proliferation assay using CCK- 8 was performed 24 and $48 \mathrm{~h}$ after transfection. The proliferation of RPMI- 8226 cells was hampered by PGC-1 $\alpha$ or ERR- $\alpha$ inhibition at 24 and $48 \mathrm{~h}$ after transfection.

of cells were observed to have crossed the membrane of the Transwell chamber. RPMI-8226 cells transfected with siRNA targeting PGC-1 $\alpha$ or ERR- $\alpha$ inhibited HUVEC migration and the counts of the migratory HUVECs reduced.

Suppression of PGC-1 $\alpha$ reduces GLUT-4 expression in MM. Myeloma cells exhibit upregulated expression of GLUT-4, which is necessary for glucose consumption, lactate production, growth and viability (3). We sought to determine whether PGC-1 $\alpha$ plays a role in the regulation of GLUT-4 in MM. As shown in Fig. 4, suppression of PGC-1 $\alpha$ led to decreased GLUT-4 expression in the RPMI-8226 cells.

Inhibition of $P G C-1 \alpha$ results in growth and proliferation defects. Cells transfected with siPGC- $1 \alpha$ or siERR- $\alpha$ were found to grow more slowly than the control cells. Similarly, the results of the CCK-8 assay also showed that proliferation of RPMI-8226 cells was diminished by siPGC- $1 \alpha$ or siERR- $\alpha$ (Fig. 5), indicating that PGC-1 $\alpha$ or ERR- $\alpha$ inhibition hampers the proliferation of myeloma cells.

\section{Discussion}

MM is a hematological malignancy characterized by the aberrant expression of malignant plasma cells within the bone marrow (14). A number of studies have shown that increased microvessel density (MVD) correlates with disease state, suggesting that increased bone marrow angiogenesis is important in myeloma progression (15). Other studies indicate that a high rate of glucose consumption beyond that necessary for ATP synthesis is exhibited by transformed cells including MM cells (16). This phenomenon has been further confirmed by F-18 fluorodeoxyglucose positron emission tomography-computed tomography (F-18-FDG PET-CT) scanning. Studies have shown that MM exhibits a high uptake rate of F-18 FDG and is positively correlated with the percentage of CD38/CD138-expressing myeloma cells in the bone marrow $(17,18)$. Moreover, Kaira et al (19) showed that F-18 FDG uptake in cancers is determined by the presence of glucose metabolism, angiogenesis and other factors, suggesting that MM with high rates of F-18 FDG uptake is most likely characterized by increased angiogenesis and glucose metabolism.

Research on the formation of new blood vessels and VEGF in particular, is a major focus of MM investigations and has led to the clinical approval of monoclonal anti-VEGF agents (20). Although these agents show significant preclinical and clinical anticancer activity, they prolong overall survival of patients for months only, after which the tumor continues to grow. Therefore, an understanding of tumor angiogenesis is still needed (20). 


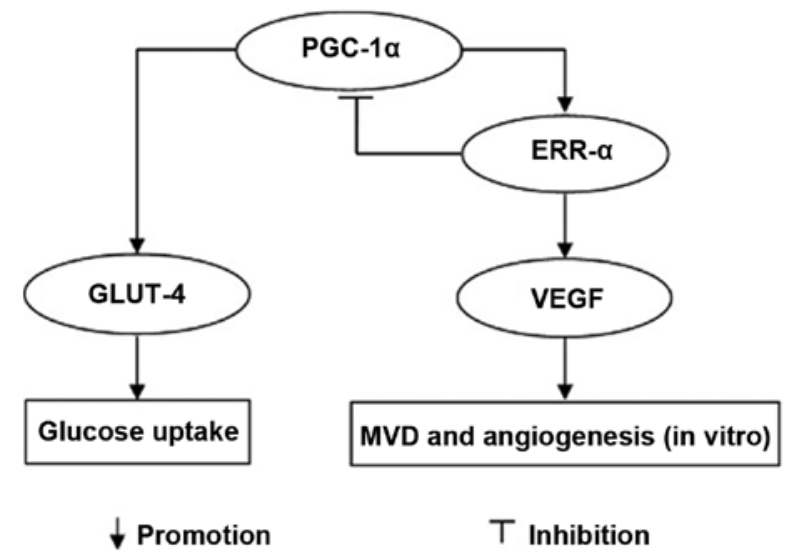

Figure 6. PGC-1 $\alpha$ connects glucose metabolism and angiogenesis in MM cells by regulating VEGF and GLUT-4. PGC-1 $\alpha$ triggers expression of ERR- $\alpha$ and GLUT-4, leading to greater glucose uptake and inducing VEGF expression and resulting in angiogenesis. ERR- $\alpha$ negatively regulates the level of PGC-1 $\alpha$; the underlying mechanism requires further exploration.

The present study demonstrated that PGC- $1 \alpha$ strongly regulated VEGF expression and angiogenesis by coactivating the orphan nuclear receptor ERR- $\alpha$, suggesting that PGC-1 $\alpha$ and ERR- $\alpha$ control a novel angiogenic pathway that delivers the needed oxygen and substrates (3). In addition, it has been suggested that activation of the ERR- $\alpha / \mathrm{PGC}-1 \alpha$ pathway increases VEGF expression and angiogenesis (21), and that the ErbB2/Neu-induced mammary tumor cells ectopically expressing PGC-1 $\alpha$ exhibit increased concentrations of the angiogenic factor VEGF compared with controls (22). Our results showed that PGC- $1 \alpha$ and ERR- $\alpha$ are upregulated in $\mathrm{MM}$ and their expression was associated with the VEGF level. They also showed that PGC- $1 \alpha$ or ERR- $\alpha$ inhibition can significantly suppress the expression of VEGF in vitro. Taken together, the results suggest that PGC- $1 \alpha$ and ERR $\alpha$, major regulators of mitochondrial function and cellular energy metabolism, also play an important role in the regulation of VEGF and angiogenesis, not only in normal cells and tissues but also in MM. This also illustrates that only targeting the classical pathways involved in angiogenesis could probably not achieve the goal of anti-angiogenesis. Indeed, lenalidomide and other similar agents show limited efficacy in the treatment of MM, and this further supports the complexity of tumor angiogenesis. Targeting PGC- $1 \alpha$ and/or ERR- $\alpha$ may improve the anti-angiogenesis effect of agents in clinical use.

Deregulation of glycolytic metabolism is another feature of MM (23). This observation and F-18-FDG PET results regarding $\mathrm{MM}$ suggest the reliance of myeloma on increased glucose consumption and glycolysis $(24,25)$. Glucose is involved in generating $\mathrm{NADH}$ and $\mathrm{FADH}$, which contribute to maintaining the integrity of mitochondria (26). Glucose also prevents the release of cytochrome $c$ by maintaining the interaction between hexokinase II and mitochondria $(27,28)$. In addition to generating energy-related chemicals, such as ATP, glucose also provides biosynthetic intermediates for lipid and nucleotide synthesis and plays a role in the regulation of several factors associated with cell death (i.e., Mcl-1, Bcl-2associated death promoter protein) (29-32). The contribution of the glycolytic phenotype to increased resistance to apop- tosis-inducing agents $(33,34)$ supports the benefits of targeting glucose consumption.

Few studies have focused on determining the biological and molecular role of GLUT activation in tumors, knowledge that could facilitate the identification of potential therapeutic targets. McBrayer et al (3) performed gene expression profiling studies to identify deregulated GLUT family members in MM and demonstrated that myeloma cells exhibit reliance on constitutively cell surface-localized GLUT-4 for basal glucose consumption, maintenance of Mcl-1 expression, growth and survival. It can, thus, be concluded that GLUT-4 is highly important to the MM proliferation and progression. GLUT-4 and its regulation controlled by other factors are well studied in normal cells. However, the pathway involved in regulating the expression of GLUT-4 is rarely studied in cancer, especially in MM. Our investigation of the interaction between MM cell proliferation and GLUT-4 activity delineates a pathway linking GLUT-4 activity with the aberrant expression of PGC-1 $\alpha$. PGC-1 $\alpha$ inhibition can decrease GLUT-4 expression and results in hampered proliferation of RPMI-8226 cells. However, by silencing ERR- $\alpha$ expression, PGC- $1 \alpha$ expression is as much as 7-fold increased and GLUT-4 expression is also increased, suggesting that ERR- $\alpha$ is a repressor of PGC-1 $\alpha$ and that the latter is associated with GLUT-4 regulation, probably by enhancing transcription of the GLUT-4 gene (Fig. 6).

ERR- $\alpha$ is an orphan member of the nuclear receptor superfamily of transcription factors whose activity is regulated by the expression level and/or activity of its obligate co-regulator, PGC-1 $\alpha$. Under normal physiological conditions, and in response to different environmental stimuli, the ERR- $\alpha /$ PGC-1 $\alpha$ complex is involved in regulating metabolic homeostasis under conditions of high energy demand in brown adipocytes, proliferating $\mathrm{T}$ cells and muscle. Notably, increased expression and activity of the ERR- $\alpha / \mathrm{PGC}-1 \alpha$ axis have also been shown to correlate with unfavorable clinical outcomes in both breast and ovarian tumors (35). However, little is known about the role of ERR- $\alpha /$ PGC- $1 \alpha$ in hematological malignances, particularly in MM.

In conclusion, our results demonstrate that PGC-1 $\alpha$ and ERR- $\alpha$ are both upregulated in MM and human myeloma RPMI-8226 cells. Furthermore, this upregulation affects the in vitro expression of GLUT-4 and the angiogenesis in MM by increasing VEGF expression. Suppression of PGC-1 $\alpha$ impairs the proliferation of RPMI-8226 cells, although not to a very great extent. Targeting PGC-1 $\alpha$ may provide another effective and probably more potent way to curb the increased needs of glucose and angiogenesis in MM.

\section{References}

1. Munshi NC: Plasma cell disorders: an historical perspective. Hematology Am Soc Hematol Educ Program: 297, 2008.

2. Ria R, Reale A, De Luisi A, Ferrucci A, Moschetta M and Vacca A: Bone marrow angiogenesis and progression in multiple myeloma. Am J Blood Res 1: 76-89, 2011.

3. McBrayer SK, Cheng JC, Singhal S, Krett NL, Rosen ST and Shanmugam M: Multiple myeloma exhibits novel dependence on GLUT4, GLUT8, and GLUT11: implications for glucose transporter-directed therapy. Blood 119: 4686-4697, 2012.

4. Stocks T, Rapp K, Bjorge T, et al: Blood glucose and risk of incident and fatal cancer in the metabolic syndrome and cancer project (me-can): analysis of six prospective cohorts. PLoS Med 6: e1000201, 2009. 
5. Vacca A, Ribatti D, Roccaro AM, Frigeri A and Dammacco F Bone marrow angiogenesis in patients with active multiple myeloma. Semin Oncol 28: 543-550, 2001.

6. Giatromanolaki A, Bai M, Margaritis D, et al: Hypoxia and activated VEGF/receptor pathway in multiple myeloma. Anticancer Res 30: 2831-2836, 2010.

7. Sun CY, Hu Y, Huang J, et al: Brain-derived neurotrophic factor induces proliferation, migration, and VEGF secretion in human multiple myeloma cells via activation of MEK-ERK and PI3K/AKT signaling. Tumour Biol 31: 121-128, 2010.

8. Puigserver $\mathrm{P}, \mathrm{Wu} \mathrm{Z}$, Park $\mathrm{CW}$, Graves R, Wright $\mathrm{M}$ and Spiegelman BM: A cold-inducible coactivator of nuclear receptors linked to adaptive thermogenesis. Cell 92: 829-839, 1998.

9. Handschin $\mathrm{C}$ and Spiegelman BM: Peroxisome proliferator-activated receptor $\gamma$ coactivator 1 coactivators, energy homeostasis, and metabolism. Endocr Rev 27: 728-735, 2006.

10. Puigserver P and Spiegelman BM: Peroxisome proliferatoractivated receptor- $\gamma$ coactivator $1 \alpha($ PGC- $1 \alpha)$ : transcriptional coactivator and metabolic regulator. Endocr Rev 24: 78-90, 2003.

11. Finck BN and Kelly DP: PGC-1 coactivators: inducible regulators of energy metabolism in health and disease. J Clin Invest 116: 615-622, 2006.

12. Arany Z, Foo SY, Ma Y, et al: HIF-independent regulation of VEGF and angiogenesis by the transcriptional coactivator PGC-1 $\alpha$. Nature 451: 1008-1012, 2008.

13. Lu J, Zhang K, Chen S and Wen W: Grape seed extract inhibits VEGF expression via reducing HIF-1 $\alpha$ protein expression. Carcinogenesis 30: 636-644, 2009.

14. Otjacques E, Binsfeld M, Noel A, Beguin Y, Cataldo D and Caers J: Biological aspects of angiogenesis in multiple myeloma. Int J Hematol 94: 505-518, 2011.

15. Nikhil C and Kenneth C (eds): Advances in Biology and Therapy of Multiple Myeloma. Volume 1: Basic Science. Springer, New York, NY, 2012.

16. Adekola K, Rosen ST and Shanmugam M: Glucose transporters in cancer metabolism. Curr Opin Oncol 24: 650-654, 2012.

17. Ak I and Gulbas Z: F-18 FDG uptake of bone marrow on PET/CT scan: its correlation with CD38/CD138 expressing myeloma cells in bone marrow of patients with multiple myeloma. Ann Hematol 90: 81-87, 2011.

18. Bartel TB, Haessler J, Brown TL, et al: F18-fluorodeoxyglucose positron emission tomography in the context of other imaging techniques and prognostic factors in multiple myeloma. Blood 114: 2068-2076, 2009

19. Kaira K, Endo M, Abe M, et al: Biologic correlation of $2-\left[{ }^{18} \mathrm{~F}\right]$-fluoro-2-deoxy-D-glucose uptake on positron emission tomography in thymic epithelial tumors. J Clin Oncol 28 : 3746-3753, 2010.

20. Podar K and Anderson KC: Emerging therapies targeting tumor vasculature in multiple myeloma and other hematologic and solid malignancies. Curr Cancer Drug Targets 11: 1005-1024, 2011.
21. Klimcakova E, Chenard V, McGuirk S, et al: PGC-1 $\alpha$ promotes the growth of ErbB2/Neu-induced mammary tumors by regulating nutrient supply. Cancer Res 72: 1538-1546, 2012.

22. Altenberg B and Greulich KO: Genes of glycolysis are ubiquitously overexpressed in 24 cancer classes. Genomics 84 1014-1020, 2004.

23. Durie BG, Waxman AD, D'Agnolo A and Williams CM: Wholebody ${ }^{18} \mathrm{~F}$-FDG PET identifies high-risk myeloma. J Nucl Med 43: 1457-1463, 2002

24. Zhang K, Lu J, Mori T, et al: Baicalin increases VEGF expression and angiogenesis by activating the ERR $\alpha / \mathrm{PGC}-1 \alpha$ pathway. Cardiovasc Res 89: 426-435, 2011.

25. Bredella MA, Steinbach L, Caputo G, Segall G and Hawkins R: Value of FDG PET in the assessment of patients with multiple myeloma. AJR Am J Roentgenol 184: 1199-1204, 2005

26. Gendron MC, Schrantz N, Metivier D, et al: Oxidation of pyridine nucleotides during Fas- and ceramide-induced apoptosis in Jurkat cells: correlation with changes in mitochondria, glutathione depletion, intracellular acidification and caspase 3 activation. Biochem J 353: 357-367, 2001.

27. Mathupala SP, Ko YH and Pedersen PL: Hexokinase II: cancer's double-edged sword acting as both facilitator and gatekeeper of malignancy when bound to mitochondria. Oncogene 25: 4777-4786, 2006

28. Gottlob K, Majewski N, Kennedy S, Kandel E, Robey RB and Hay N: Inhibition of early apoptotic events by Akt/PKB is dependent on the first committed step of glycolysis and mitochondrial hexokinase. Genes Dev 15: 1406-1418, 2001.

29. DeBerardinis RJ, Mancuso A, Daikhin E, et al: Beyond aerobic glycolysis: transformed cells can engage in glutamine metabolism that exceeds the requirement for protein and nucleotide synthesis. Proc Natl Acad Sci USA 104: 19345-19350, 2007.

30. Zhao Y, Altman BJ, Coloff JL, et al: Glycogen synthase kinase $3 \alpha$ and $3 \beta$ mediate a glucose-sensitive antiapoptotic signaling pathway to stabilize Mcl-1. Mol Cell Biol 27: 4328-4339, 2007.

31. Danial NN, Gramm CF, Scorrano L, et al: BAD and glucokinase reside in a mitochondrial complex that integrates glycolysis and apoptosis. Nature 424: 952-956, 2003.

32. Rathmell JC, Fox CJ, Plas DR, Hammerman PS, Cinalli RM and Thompson CB: Akt-directed glucose metabolism can prevent Bax conformation change and promote growth factor-independent survival. Mol Cell Biol 23: 7315-7328, 2003.

33. Gatenby RA and Gillies RJ: Why do cancers have high aerobic glycolysis? Nat Rev Cancer 4: 891-899, 2004.

34. Plas DR and Thompson CB: Cell metabolism in the regulation of programmed cell death. Trends Endocrinol Metab 13: 75-78, 2002.

35. Chang CY and McDonnell DP: Molecular pathways: the metabolic regulator estrogen-related receptor $\alpha$ as a therapeutic target in cancer. Clin Cancer Res 18: 6089-6095, 2012. 